\title{
Measurement of Extraction and Absorption Parameters in GaN-based Photonic-crystal Light- emitting Diodes
}

\section{Citation}

Matioli, Elison, Blaise Fleury, Elizabeth Rangel, Evelyn Hu, James Speck, and Claude Weisbuch. 2010. Measurement of extraction and absorption parameters in GaN-based photonic-crystal light-emitting diodes. Journal of Applied Physics 107(5): 053114.

\section{Published Version}

doi:10.1063/1.3309837

\section{Permanent link}

http://nrs.harvard.edu/urn-3:HUL.InstRepos:11005280

\section{Terms of Use}

This article was downloaded from Harvard University's DASH repository, and is made available under the terms and conditions applicable to Other Posted Material, as set forth at http:// nrs.harvard.edu/urn-3:HUL.InstRepos:dash.current.terms-of-use\#LAA

\section{Share Your Story}

The Harvard community has made this article openly available.

Please share how this access benefits you. Submit a story.

\section{Accessibility}




\title{
Measurement of extraction and absorption parameters in GaN-based photonic-crystal light-emitting diodes
}

\author{
Elison Matioli, ${ }^{1, a)}$ Blaise Fleury, ${ }^{1}$ Elizabeth Rangel, ${ }^{1}$ Evelyn $\mathrm{Hu},{ }^{1,2}$ James Speck, ${ }^{1}$ and \\ Claude Weisbuch ${ }^{1,3}$ \\ ${ }^{1}$ Department of Materials, University of California, Santa Barbara, California 93106-5050, USA \\ ${ }^{2}$ School of Engineering and Applied Physics, Harvard University, Cambridge, Massachusetts 02138, USA \\ ${ }^{3}$ Laboratoire de Physique de la Matière Condensée, Ecole Polytechnique, CNRS, 91128 Palaiseau, \\ France
}

(Received 10 November 2009; accepted 12 January 2010; published online 12 March 2010)

\begin{abstract}
The light extraction efficiency of photonic-crystal $(\mathrm{PhC})$ light-emitting diodes (LEDs) relies on the competition between the $\mathrm{PhC}$ extraction and dissipation mechanisms of the guided light within the LED. This work presents the experimental determination of the PhC extraction length of each guided mode and the absorption coefficient of the active region (AR) and quantum wells (QWs) from the observation of the LED far-field emission using a high-resolution angle-spectrum-resolved measurement. The angular and spectral linewidths of the extracted guided modes reveal, depending on the spectral range, the modal extraction length of the $\mathrm{PhCs}$, the $\mathrm{AR}$ absorption length, or a combination of both. Modes with a high confinement with the QWs presented a shorter absorption length compared with their extraction length by a shallow surface $\mathrm{PhC}$ (95-nm-deep), meaning that the $\mathrm{AR}$ absorption was a more efficient mechanism than the $\mathrm{PhC}$ extraction. The measured modal extraction length of the shallow surface $\mathrm{PhC}$ varied in the range of 55-120 $\mu \mathrm{m}$, which determines the minimum dimensions of the device and the maximum acceptable dissipation length for an efficient extraction of the guided light by the PhCs. This paper presents also a discussion on the $\mathrm{PhC}$ designs that yield $\mathrm{PhC}$ extraction lengths shorter than other dissipation lengths, a fundamental requirement for high-efficiency PhC LEDs. The same technique was also applied to estimate the absorption coefficient of the InGaN-based QWs, and can be extended to experimentally determine losses by metallic layers from electrical contacts or other dissipation mechanisms, which are parameters of interest to a broader class of optoelectronic devices, not only PhC LEDs.
\end{abstract}

(C) 2010 American Institute of Physics. [doi:10.1063/1.3309837]

\section{INTRODUCTION}

Photonic crystals (PhCs) have, from the beginning, been evoked as a way to realize high-efficiency light sources. ${ }^{1}$ The early concept of controlling spontaneous light emission by an omnidirectional three-dimensional (3D) photonic bandgap (PBG) has had limited success in devices due to practical difficulties, such as achieving highly ordered 3D PhCs (Ref. 2) and proper positioning of the emitting species within the structure. ${ }^{2-4}$ The concept has been most convincingly demonstrated in membrane-supported two-dimensional (2D) $\mathrm{PhCs}$. However, the strong reduction in spontaneous radiative emission rate for photons within the PBG requires one to rely on low temperature operation to diminish the competing nonradiative recombination and maintain a good internal quantum efficiency. ${ }^{3,5,6}$ In contrast, a growing body of work has revealed the efficacy of $2 \mathrm{D} \mathrm{PhC}$ patterning of GaN-based light-emitting diodes (LEDs) to achieve greater light extraction, and to control the directionality of the emitted light. ${ }^{7-11}$ In these cases, the $2 \mathrm{D} \mathrm{PhC}$ operates mostly outside the PBG spectral range, acting as a diffraction grating placed directly on the light source. High light extraction efficiency requires a structure design that optimizes the interaction of the guided modes with the PhCs through an appropriate selection of the

${ }^{\text {a)} E l e c t r o n i c ~ a d d r e s s: ~ e l i s o n . m a t i o l i @ ~ p o l y t e c h n i q u e . o r g . ~}$
LED and PhC parameters (thickness of layers, depth of the $\mathrm{PhCs}$, position of the quantum wells (QWs), $\mathrm{PhC}$ period, and fill factor), which will translate into shorter characteristic extraction length of the guided modes compared with any dissipation length, such as absorption in the QWs and doped layers and, depending on the precise LED structure, dissipation losses from contacting materials. PhC LED optimization has relied so far on simulations of $\mathrm{PhC}$ extraction length and on some modeling of the losses. ${ }^{12-14}$ The $\mathrm{PhC}$ enhancement in light extraction is commonly assessed by output power measurements, which only reveal the global effect of the $\mathrm{PhCs}$ on the total light extraction of the guided light inside the LED. The experimental determination of the PhC extraction length and other dissipation lengths presented here indicates the minimum LED chip size that allows for extraction of all guided light. In addition, determination of these length scales reveals the competition between the $\mathrm{PhC}$ extraction and the absorption mechanisms.

This paper presents an experimental technique that, from a simple analysis, has the ability to provide extraction lengths of guided modes, absorption of QWs, and active region (AR). The variation in these parameters with photon energy is measured, their dependence on the structure parameters is evaluated and the design rules for PhC LEDs are discussed based on a deep understanding of the entire device from the individual modal behavior analysis. 


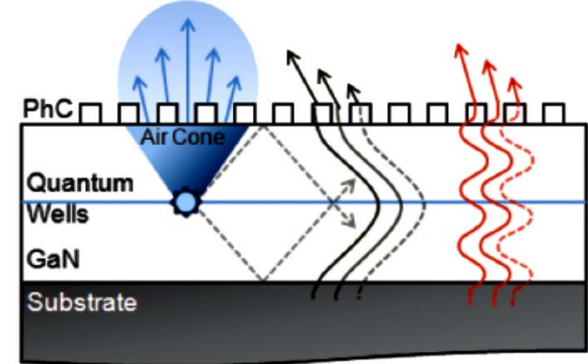

(a)

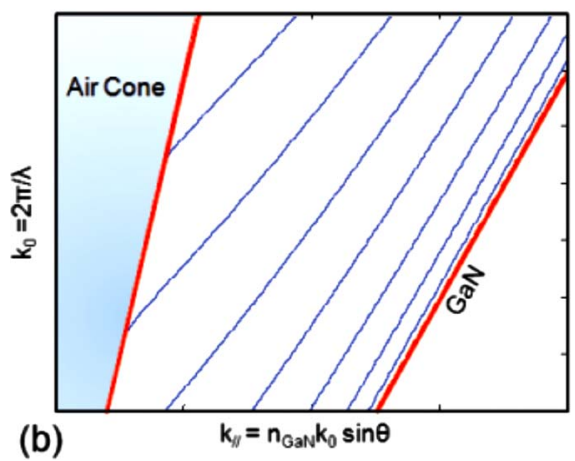

FIG. 1. (Color online) Schematics of the formation of guided modes in a GaN-based PhC LEDs. (a) Schematics of the directly emitted light and the $\mathrm{PhC}$ extraction of guided modes as they propagate inside the LED. (b) Dispersion relation of the TE-polarized guided modes in a simple waveguide showing that for a given wavelength $\lambda$, a finite set of modes is supported by the waveguide.

\section{PHOTONIC CRYSTALS AS AN INTERNAL PROBE}

Diffraction properties of PhC LEDs are quite different from those observed in usual 3D crystals. In the latter, the diffracted beam from a monochromatic light source (or a plane-wave) impinging on a perfect and infinite (or semiinfinite) crystal is also monochromatic and occurs at a welldefined angle given by the diffraction laws. As the wavelength of the light source broadens and/or the crystal is no longer perfect or infinite, the diffracted beam acquires a nonzero linewidth, both spatially and spectrally. This phenomenon can be used to either analyze the light source (spectrometry) or the quality of the crystal (x-ray diffraction).

From simple considerations, it would be natural to expect, in 2D PhC LEDs, broad diffracted peaks due to the extended light source nature of the QWs and to the omnidirectional incidence of light on the diffracting 2D PhC. However, angle-resolved measurements reveal very well-defined diffracted peaks, ${ }^{8}$ much sharper than the QW emission linewidth. This comes from the fact that the extended source emitting light inside a semiconductor slab excites a finite set of guided modes that propagate through constructive interferences at well-defined, discrete angles with the waveguide interface [Fig. 1(a)]. The dispersion relation of the guided modes inside a waveguide [Fig. 1(b)] implies that, for a given wavelength, a finite set of modes is supported by the waveguide.

If we then consider a diffracting grating (or $\mathrm{PhC}$ ) on the surface of this waveguide, each guided mode will be diffracted out of the waveguide at a well-defined angle. The linewidth of each diffracted guided mode is related to its extraction length by the grating, therefore the measurement of the modal linewidth serves as a probe of the PhC modal extraction. Thus, each guided mode acts just like one separate monochromatic source sampling a finite length of the $\mathrm{PhC}$, equal to its own extraction length, which determines the linewidth of this diffracted mode.

The guided modes also probe absorption mechanisms, such as QW reabsorption, which again determines the linewidth of the diffracted mode. Due to the spectral width of the source [full width at half maximum (FWHM) of $\sim 20 \mathrm{~nm}$ ], the various excited modes can monitor the dependence of the QW reabsorption on the photon energy close to the QW absorption edge. More generally, the PhCs can be used as an internal probe to measure optical properties of specific layers in optoelectronic devices such as absorption of the QWs, metallic or semiconductor layers.

\section{MODEL TO DETERMINE THE MODAL EXTRACTION LENGTH}

In a simple planar structure with embedded QWs, such as planar LEDs, only the small fraction of the light emitted from the QWs that falls within the air cone is emitted to air [Fig. 1(a)]. The remainder stays inside the structure as guided modes due to total internal reflection on the semiconductor/air interfaces, otherwise stated as modes having in-plane wavevectors larger than the air wavevector [Fig. 1(b)]. The guided light propagating inside the LED structure is composed of a finite set of guided modes, each represented by a plane wave $\vec{E}^{n}(\vec{r})=\vec{E}_{o}^{n} e^{i \vec{k}_{n} \vec{r}}$, where $\vec{k}_{n}=\vec{k}_{\|_{n}}+\vec{k}_{z_{n}}$ and $n$ labels each mode (the index $n$ will be abandoned in the remainder of the paper for simplicity). The spatial modulation of index of refraction in real space of the $\mathrm{PhCs}$, described in reciprocal space by a reciprocal vector $\vec{G}$, outcouples the guided modes by changing their in-plane wavevector as $\vec{k}_{\|, m}=\vec{k}_{\|}+m \vec{G}$, where $m$ is an integer. The infinite set of harmonics diffracted by the $\mathrm{PhCs}$ form a single mode (Bloch mode) which is an electromagnetic solution of this periodic medium and is represented by $\vec{E}(\vec{r})=\sum_{m} \vec{E}_{m} e^{i\left(\vec{k}_{\|, m} \vec{r}_{\mid+1}+k_{z} z\right)}$, where $\vec{r}_{\|}=x \vec{e}_{x}+y \vec{e}_{y}$ and $\vec{k}_{\|}=k_{x} \vec{e}_{x}+k_{y} \vec{e}_{y}$ are the in-plane spatial vector and wavevector, respectively, and $z$ and $k_{z}$ are their out-ofplane components.

The diffracted in-plane harmonics that fall inside the air cone (in other words, satisfying the relationship $\left|\vec{k}_{\|, m}\right|<k_{0}$ ) radiate to air and introduce an imaginary component $k^{\prime \prime}$ to the Bloch mode wavevector. The energy of such leaky mode decreases exponentially as it propagates in the structure from the emission point as $I=|\vec{E}(\vec{r})|^{2}=\left|\vec{E}_{0}\right|^{2} e^{-2 k^{\prime \prime} r_{\|}}$due to diffraction to air by the PhCs. ${ }^{13}$ An extraction length for each guided mode can be defined as $L_{\text {extraction }}=1 / 2 k^{\prime \prime}$.

The effect of an exponentially decreasing intensity in real space $\left(r_{\|}\right)$can be seen in reciprocal space by the correspondent conjugate variable $k$, obtained by Fourier transformation, which results in a Lorentzian

$$
\mathcal{F}[I](k)=4 k^{\prime \prime}\left|\vec{E}_{0}\right|^{2} /\left(4 k^{\prime \prime 2}+k^{2}\right),
$$

whose FWHM, designated here $\Delta k_{\|}$, is equal to $4 k^{\prime \prime}$ which is related to $L_{\text {extraction }}$ as 


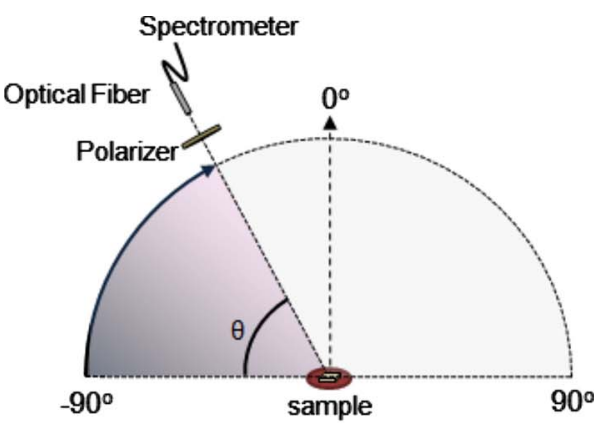

(a)

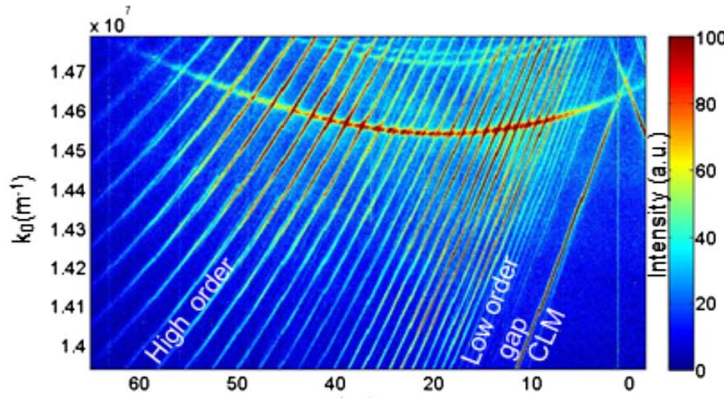

(b)

Angle $\theta$

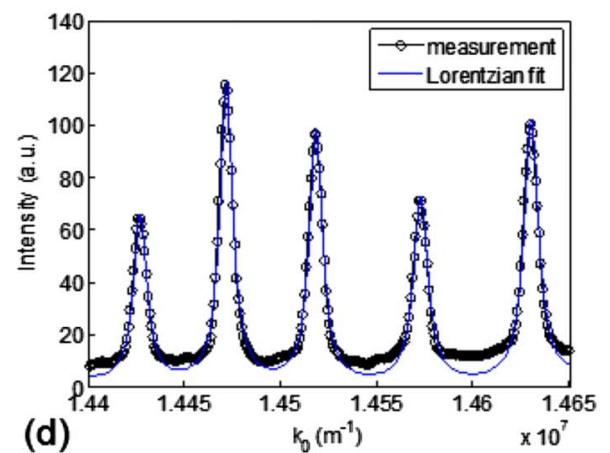

(d) (c)

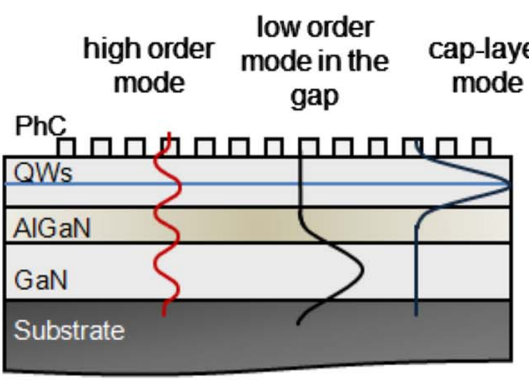

FIG. 2. (Color online) (a) Schematics of the high resolution angle-resolved setup. (b) High-resolution angle-resolved measurement where the wavelength $\lambda$ was converted into $k_{0}=2 \pi / \lambda$. The sharp lines correspond to the extracted guided modes by the PhCs. (c) Schematics of the sideview of the PhC LED structure along with the guided modes supported: cap-layer mode (CLM), low order mode in the gap and high order mode. (d) Cross section of the angle-resolved measurement at a given angle (circles) along with the theoretical Lorentzian-shaped fit (solid line).

$$
\mathrm{FWHM}=\Delta k_{\|}=2 / L_{\text {extraction }} \text {. }
$$

The extraction length of all guided modes is determined from the measurement of $\Delta k_{\|}$, which is assessed from the far-field emission of the LED. Other scattering and dissipation processes, due to imperfections in the LED and in the $\mathrm{PhCs}$, reabsorption in the QWs as well as in doped or metallic layers broaden the FWHM of the extracted modes. The measured decay length of the mode should now be called the extinction length $L_{\text {extinction }}=1 / 2\left(k_{s}^{\prime \prime}+k_{\text {phc }}^{\prime \prime}+k_{a}^{\prime \prime}\right)$, where $k_{s}^{\prime \prime}$ and $k_{a}^{\prime \prime}$ correspond, respectively, to the scattering and absorption components of $k^{\prime \prime}$ and $k_{\mathrm{phc}}^{\prime \prime}$ corresponds to the PhC diffraction.

\section{MEASUREMENT OF MODAL EXTRACTION LENGTH}

The measurement of the extracted guided modes in the reciprocal space is done by high-resolution angle-resolved electroluminescence, similar to the one described by David et al. ${ }^{8}$ however the spatial and spectral resolution have been considerably increased to properly measure the FWHM of the guided modes. The far-field spectrum of the LED under electric bias is collected, for all angles $\theta$ from $-90^{\circ}$ to $90^{\circ}$, by a $100 \mu \mathrm{m}$ aperture optical fiber placed on a $21 \mathrm{~cm}$ radius rotating arm after a proper selection of the TE or TM modes by a polarizer placed in front of the optical fiber. No lenses are used to increase the fiber collection angle which would be detrimental to the setup resolution. The measured spectra were sent through the optical fiber to a spectrometer (Triax 550-Horiba Jobin-Yvon) with a 0.1 and $0.2 \mathrm{~mm}$ entrance slit (further explanation on the choice of the spectrometer slit is given later) and a $1800 \mathrm{~g} / \mathrm{mm}$ diffraction grating. A schematic of the rotating setup is shown in Fig. 2(a).

The collection of all the spectra versus angle, after a proper conversion of axis as $(\theta, \lambda) \rightarrow\left[k_{\|, m}=k_{0} \sin (\theta), k_{0}\right]$, reveals the dispersion relation of the extracted guided modes ${ }^{8}$ $\left(k_{\|, m}\right.$ is the parallel component of the diffracted wavevector and $\left.k_{0}=2 \pi / \lambda\right)$. Each mode can be labeled by its effective index of refraction $n_{\text {eff }}=k_{\|} / k_{0}$ and its extraction length can be obtained from a measurement of its FWHM and Eq. (2). The result of the angle-resolved measurement along the $\Gamma \mathrm{K}$ direction of the PhCs is shown in Fig. 2(b). The sharp oblique lines correspond to the $\mathrm{PhC}$ extraction of modes guided in the LED structure [guided modes represented in Fig. 2(c)].

The GaN-based LEDs measured in this work were grown by metalorganic chemical vapor deposition on a sapphire substrate. The LED structure consists of a $\sim 3$ - $\mu$ m-thick n-GaN followed by a 650-nm-thick $\mathrm{Al}_{0.16} \mathrm{Ga}_{0.84} \mathrm{~N}$ confining layer and a 550 -nm-thick AR comprised of a $\mathrm{n}-\mathrm{GaN}$ layer, $6 \mathrm{x}-\mathrm{InGaN}$ QWs emitting at $\lambda$ $=445 \mathrm{~nm}$ and a p-GaN layer [Fig. 2(b)]. The 95-nm-deep $\mathrm{PhC}$ with triangular lattice and $28 \%$ fill factor was patterned on the LED top surface with period of $200 \mathrm{~nm}$ [Fig. 2(c)]. The device size was $800 \times 800 \mu \mathrm{m}^{2}$ and the current was injected through a circular p-contact (diameter of $100 \mu \mathrm{m}$ ) in the center of the device to separate the metal absorption from the $\mathrm{PhC}$ extraction of the guided modes.

The $\mathrm{Al}_{0.16} \mathrm{Ga}_{0.84} \mathrm{~N}$ layer confines one low order mode in the top layer which has a high interaction with both the $\mathrm{PhCs}$ and the QWs. ${ }^{12}$ This mode is referred to as the cap-layer mode (CLM). The bright lines crossing at $0^{\circ}$ in Fig. 2(b) correspond to the extraction of the CLM represented by the 
(a)
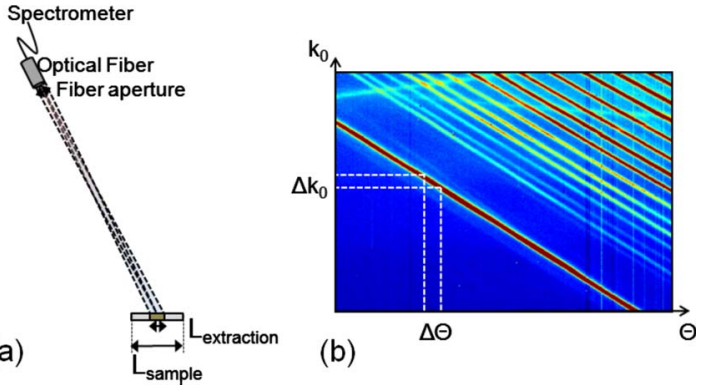

FIG. 3. (Color online) (a) Schematics of the setup angular resolution showing its limitation due to the fiber aperture and sample size. (b) Dispersion relation of the extracted modes by the PhCs indicating the effect of the setup angular aperture $\Delta \theta$ on $\Delta k_{0}$.

blue mode in Fig. 2(c). The gap between the extracted modes and the CLM in Fig. 2(b) corresponds to the low order modes guided in the GaN layer below the $\mathrm{Al}_{0.16} \mathrm{Ga}_{0.84} \mathrm{~N}$, which are neither well excited by the QWs nor extracted by the PhCs [represented in black in Fig. 2(c)]. The modes diffracted at higher angles are high order modes represented in red in Fig. 2(c).

Figure 2(d) shows a cross section of the angle-resolved measurement (blue curve) at a given angle where each peak corresponds to a different guided mode extracted by the $\mathrm{PhCs}$ and the red curve is its theoretical Lorentzian-shaped fit, as predicted by Eq. (1) and explained in more details in the following paragraphs. The precision of the measurement of $\Delta k_{\|}$depends largely on the angular resolution of the angleresolved setup. The collection solid angle of the optical fiber which is determined by its $100 \mu \mathrm{m}$-aperture at a distance of $21 \mathrm{~cm}$ to the sample limits the angular resolution of the setup to $\Delta \theta \sim 0.027^{\circ}$. The resolution is degraded when the sample size is taken into account. Even though the sample is $800 \mu \mathrm{m}$-large, its effective length for the extraction of the guided modes is $L_{\text {extraction, }}$ which can be theoretically estimated (and measured in this paper) to be on the order of $\sim 100 \mu \mathrm{m}$. Hence, $\Delta \theta$ is increased to $\sim 0.055^{\circ}$ when the effective length of the sample is considered [Fig. 3(a)], which limits the proper measurement of $\Delta k_{\|}$. The resolution can be increased by either using a fiber with smaller diameter or placing the fiber further away from the sample, which in either case would considerably reduce the collected signal.

A limiting factor in the spectral resolution is the spectrometer slit size. The reduction in the slit size increases the spectral resolution but reduces the amount of signal measured by the spectrometer; thus requiring a higher integration time in the measurement. Two different slit sizes were used in the measurements presented in this paper. A slit size of 0.2 $\mathrm{mm}$ was used for the measurement of the extraction length of all the guided modes, shown in Fig. 2(b), because of the higher range of angles needed for this measurement. A slit of $0.1 \mathrm{~mm}$ was used for the measurement of the extinction length of the CLM [shown later in Fig. 6(a)], where the smaller range of angles allowed for a longer integration time.

To overcome the limited angular resolution of this setup, we took advantage of the higher spectral resolution of the spectrometer to measure the FWHM of the modes along $k_{0}$ [ordinate of the measurement shown in Fig. 2(b)], designated

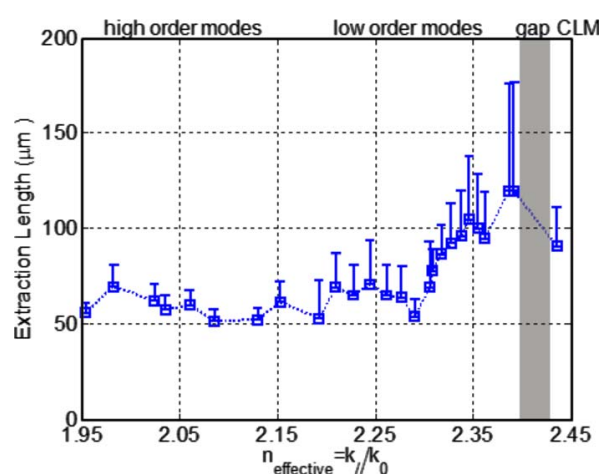

FIG. 4. (Color online) Extraction length $L_{\text {extraction }}$ as a function of the effective index of the guided modes.

$\Delta k_{0}$, which can be later converted to $\Delta k_{\|}$using the dispersion relation of the guided modes: $\Delta k_{\|}=n_{\text {eff }} \Delta k_{0}$. Thus, the extraction length is

$$
L_{\text {extraction }}=2 /\left(n_{\text {eff }} \Delta k_{0}\right) \text {. }
$$

The $\Delta k_{0}$ for all the modes at $k_{0} \sim 1.43 e 7 \mathrm{~m}^{-1}$ was measured from the cross section of the angle-resolved measurement shown in Fig. 2(d), converted to $L_{\text {extraction }}$ using Eq. (3) and the results are shown in Fig. 4.

The error bars in the measurement of extraction length in Fig. 4 were determined from an estimation of the error due to the angular aperture $\Delta \theta$ of the optical fiber (as discussed previously), which broadens the measured value of $\Delta k_{0}$ due to the dispersion of each guided mode $(\partial k / \partial \theta)_{n}$, where $n$ stands for the different modes [Fig. 3(b)]. The error bars were estimated from the convolution of a Lorentzian with a window of length $\omega_{n}=(\partial k / \partial \theta)_{n} \Delta \theta$. The broadening of the measured value of $\Delta k_{0}$ due to the spectrometer slit was measured with an Hg lamp and was also included in the error bars.

The measured extraction length for all modes varied at $55-120 \mu \mathrm{m}$, which matches the theoretical predictions, as will be shown later.

\section{MEASUREMENT OF QUANTUM-WELL ABSORPTION}

The introduction of the 650-nm-thick lower-index $\mathrm{Al}_{0.16} \mathrm{Ga}_{0.84} \mathrm{~N}$ confining layer [Fig. 2(c)] in the structure confines very strongly one single mode, the CLM, in the 550nm-thick AR, which contains the most absorbing layers in the LED: the QWs and p-doped GaN. A simple calculation of the overlap of the CLM with the QWs $\left(\Gamma_{\mathrm{QW}}\right)$ and the p-doped $\mathrm{GaN}\left(\Gamma_{\mathrm{pGaN}}\right)$ yields $\Gamma_{\mathrm{QW}}=4 \%$ and $\Gamma_{\mathrm{pGaN}}=16 \%$, which is much higher than the overlap of any other mode with these absorbing layers. Therefore the CLM is subject to a much higher absorption in the AR than the other guided modes.

The surface $\mathrm{PhCs}$ in this structure were intentionally shallow etched (95-nm-deep) to yield a large extraction length, so that the absorption in the AR competes effectively with the PhC extraction. Since the spectral linewidth of the InGaN-based QWs is large (FWHM of $\sim 20 \mathrm{~nm}$ ), the CLM can probe the variation in the absorption as a function of the photon energy. Figure 5(a) shows a low resolution angular 


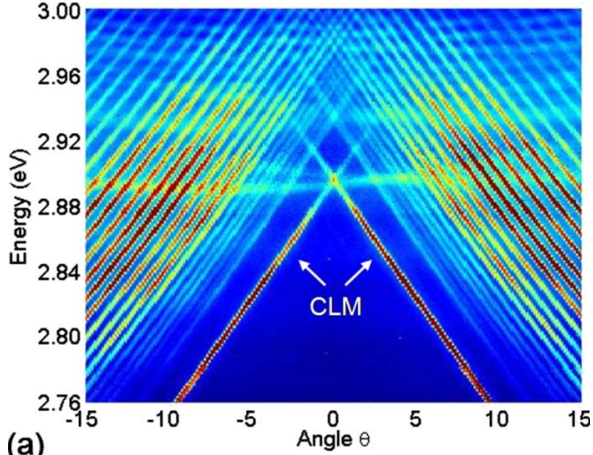

(a)

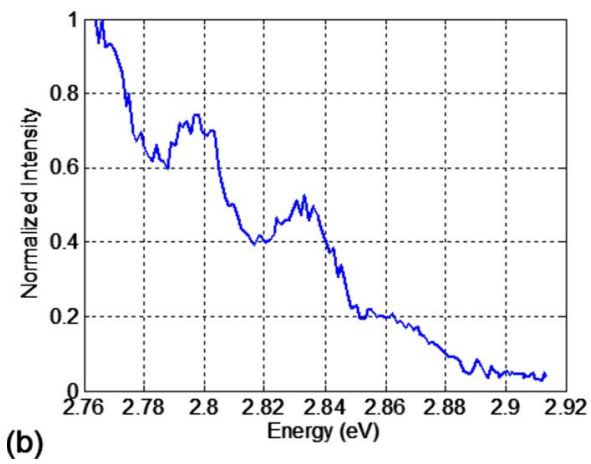

FIG. 5. (Color online) (a) Low resolution angle-resolved measurement converted into photon energy vs angle showing that the CLM disappears for energies above $2.93 \mathrm{eV}$. (b) Normalized intensity of the CLM as a function of the photon energy. The oscillations are from Fabry-Pérot interferences that modulate the intensity of the CLM.

measurement around $0^{\circ}$ after $k_{0}$ was converted into energy. It is easy to see that the CLM completely disappears above $2.93 \mathrm{eV}$, while all the other modes are still observed. The absorption of the CLM can also be seen from the plot of its intensity as a function of the photon energy, shown in Fig. 5 (b), after it was normalized by the intensity at $2.76 \mathrm{eV}$, where the absorption was assumed to be negligible, and subsequently normalized by the lineshape of the QW emission in this energy range. The intensity of the CLM decreases with energy and reaches zero at $\sim 2.92 \mathrm{eV}$. The oscillations observed in the CLM intensity come from Fabry-Pérot modulations in the cavity formed by the GaN slab. ${ }^{8}$

Assuming that, for the considered energy range, the absorption in the AR and the $\mathrm{PhC}$ diffraction are the most important loss mechanisms for the CLM, the extinction length as a function of energy is defined as $L_{\text {extinction }}(\varepsilon)$ $=1 /\left(2 k^{\prime \prime}(\varepsilon)+\alpha(\varepsilon)\right)$ [where $\alpha(\varepsilon)$ is the modal absorption coefficient per length as a function of energy] and is determined by replacing the extraction by extinction length in Eq. (3). The measured extinction length of the CLM decreases as the photon energy increases, as shown in blue in Fig. 6(a), meaning that either its extraction length decreases and/or the $\mathrm{AR}$ absorption increases as a function of energy.

The extraction length of the CLM as a function of the energy was theoretically estimated by calculating the complex in-plane wavevector of the Bloch modes supported in this structure. The calculation was based on scattering-matrix formalism ${ }^{8,15}$ considering the entire $3 \mathrm{D}$ structure with the same nominal values previously described. The imaginary component of the wavevector $k^{\prime \prime}$ was then converted into
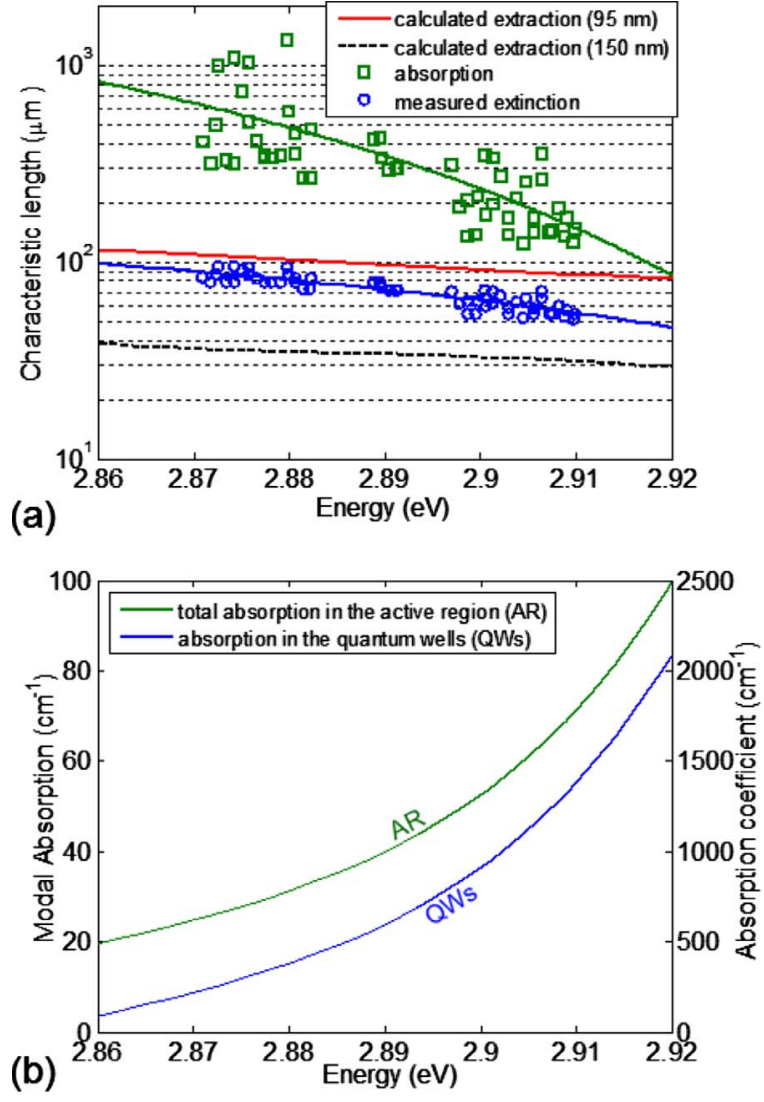

FIG. 6. (Color online) (a) Dependence of the extinction (circles), extraction (solid) and absorption lengths (squares) of the CLM on energy. The dotted curve is the CLM extraction length for a 150-nm-deep PhC, which has a better extraction than the 95-nm-deep PhC (solid). (b) Modal absorption coefficient for the CLM (left ordinate axis) and volume absorption (right ordinate axis) as a function of energy in the AR and QWs.

$L_{\text {extraction }}=1 / 2 k^{\prime \prime}$. The theoretically determined extraction length of the CLM, shown by the red curve in Fig. 6(a), also decreases with energy, however with a different slope compared with the measured extinction length. The modal absorption length in the AR can be determined from the extinction and extraction length curves by using $L_{\text {absorption }}^{-1}(\varepsilon)$ $=L_{\text {extinction }}^{-1}(\varepsilon)-L_{\text {extraction }}^{-1}(\varepsilon)$, where $L_{\text {absorption }}(\varepsilon)=1 / \alpha(\varepsilon)$. The absorption length, shown in the green in Fig. 6(a), decreases fast with energy until it becomes smaller than the $\mathrm{PhC}$ extraction length (for the 95-nm-deep PhCs) for energies above $2.92 \mathrm{eV}$, where the absorption in the AR is a more efficient mechanism than the PhC extraction. A smaller extraction length is calculated for 150-nm-deep PhCs, a more common configuration, with values between 30 and $40 \mu \mathrm{m}$ [black curve in Fig. 6(a)], well below the AR absorption length.

The modal absorption coefficient $\alpha(\varepsilon)$ determined from the AR absorption length in Fig. 6(a) is shown in the green curve in Fig. 6(b) and includes the absorptions from the QWs and p-doped GaN. The modal absorption from the p-doped $\mathrm{GaN}$ can be calculated from the $\mathrm{p}-\mathrm{GaN}$ absorption coefficient $^{16} \sim 100 \mathrm{~cm}^{-1}$ and $\Gamma_{\mathrm{pGaN}} \sim 16 \%$ which results in $\alpha_{\mathrm{pGaN}} \sim 16 \mathrm{~cm}^{-1}$. Assuming that $\alpha_{\mathrm{pGaN}}$ does not vary significantly with energy, the QW modal reabsorption coefficient can be estimated as $\alpha_{\mathrm{QW}}(\varepsilon)=\alpha(\varepsilon)-\alpha_{\mathrm{pGaN}}$ [blue curve in Fig. 6(b)].

The absorption coefficient of the 6x-InGaN QWs is es- 
timated by $\alpha_{\mathrm{QW}}(\varepsilon) / \Gamma_{\mathrm{QW}}[$ Fig. $6(\mathrm{~b})]$, assuming that $\Gamma_{\mathrm{QW}}$ does not vary significantly over the range of energies considered. While this is a direct measurement of the modal absorption in optoelectronic devices, the estimated absorption coefficient of the InGaN QWs presented here is in agreement with previous measurement of the absorption coefficient of InGaN-based QWs. ${ }^{17,18}$

\section{DISCUSSION AND CONCLUSION}

High resolution angle-resolved measurements performed on a waveguide with surface PhCs were used to measure the extraction and dissipation properties of guided modes in $\mathrm{PhC}$ LEDs. The measured modal extraction length in our LED with a shallow surface $\mathrm{PhC}$ varied in the range of 55-120 $\mu \mathrm{m}$, which determines the minimum dimensions of the PhC LED.

This technique was applied to measure the AR as well as the QW absorption of the LED. The AR absorption length was smaller than the theoretical extraction length of the CLM by the shallow surface $\mathrm{PhC}$ (95-nm-deep) for energies above $2.92 \mathrm{eV}$, meaning that, for this energy range the AR absorption is a more efficient mechanism than the $\mathrm{PhC}$ extraction. In the present case, the $\mathrm{PhCs}$ were intentionally designed to yield a poor extraction length allowing the measurement of the absorption in the AR. For high-efficiency $\mathrm{PhC}$ LEDs, the $\mathrm{PhC}$ extraction length needs to be shorter than any dissipation length. This can be achieved by several different configurations of $\mathrm{PhCs}$, such as deeper surface PhCs ( 150-200-nm-deep), thin-film LEDs with surface $\mathrm{PhCs},{ }^{10}$ or embedded PhCs. ${ }^{19,20}$ The present technique can be extended to experimentally determine losses by metallic layers from electrical contacts or other dissipation mechanisms in optoelectronic devices.

\section{ACKNOWLEDGMENTS}

This work was supported by the U.S. DOE under Project No. DE-FC26-06NT42857 and DOE-EFRC under Project No. DE-SC0001009.

${ }^{1}$ E. Yablonovitch, Phys. Rev. Lett. 58, 2059 (1987).

${ }^{2}$ I. S. Nikolaev, P. Lodahl, and W. L. Vos, Phys. Rev. A 71, 053813 (2005).

${ }^{3}$ S. Ogawa, M. Imada, S. Yoshimoto, M. Okano, and S. Noda, Science 305, 227 (2004).

${ }^{4}$ K. Aoki, D. Guimard, M. Nishioka, M. Nomura, S. Iwamoto, and Y. Arakawa, Nat. Photonics 2, 688 (2008).

${ }^{5}$ S. Noda, M. Fujita, and T. Asano, Nat. Photonics 1, 449 (2007).

${ }^{6}$ S. Noda and M. Fujita, Nat. Photonics 3, 129 (2009).

${ }^{7}$ J. J. Wierer, M. R. Krames, J. E. Epler, N. F. Gardner, M. G. Craford, J. R. Wendt, J. A. Simmons, and M. M. Sigalas, Appl. Phys. Lett. 84, 3885 (2004).

${ }^{8}$ A. David, C. Meier, R. Sharma, F. S. Diana, S. P. DenBaars, E. Hu, S. Nakamura, C. Weisbuch, and H. Benisty, Appl. Phys. Lett. 87, 101107 (2005).

${ }^{9}$ K. McGroddy, A. David, E. Matioli, M. Iza, S. Nakamura, S. DenBaars, J. S. Speck, C. Weisbuch, and E. L. Hu, Appl. Phys. Lett. 93, 103502 (2008).

${ }^{10}$ J. J. Wierer, A. David, and M. M. Megens, Nat. Photonics 3, 163, (2009).

${ }^{11}$ C.-F. Lai, C.-H. Chao, H.-C. Kuo, H.-H. Yen, C.-E. Lee, and W.-Y. Yeh, Appl. Phys. Lett. 94, 123106 (2009).

${ }^{12}$ A. David, T. Fujii, B. Moran, S. Nakamura, S. P. DenBaars, C. Weisbuch, and H. Benisty, Appl. Phys. Lett. 88, 133514 (2006).

${ }^{13}$ A. David, H. Benisty, and C. Weisbuch, J. Disp. Technol. 3, 133 (2007).

${ }^{14}$ E. Matioli, M. Iza, Y.-S. Choi, F. Wu, S. Keller, H. Masui, E. Hu, J. Speck, and C. Weisbuch, Phys. Status Solidi C 6, S675 (2009).

${ }^{15}$ S. G. Tikhodeev, A. L. Yablonskii, E. A. Muljarov, N. A. Gippius, and T. Ishihara, Phys. Rev. B 66, 045102 (2002).

${ }^{16}$ M. Kuramoto, C. Sasaoka, N. Futagawa, M. Nido, and A. A. Yamaguchi, Phys. Status Solidi A 192, 329 (2002).

${ }^{17}$ F. Renner, P. Kiesel, G. H. Dohler, M. Kneissl, C. G. Van de Walle, and N. M. Johnson, Appl. Phys. Lett. 81, 490 (2002).

${ }^{18}$ J. Kvietkova, L. Siozade, P. Disseix, A. Vasson, J. Leymarie, B. Damilano, N. Grandjean, and J. Massies, Phys. Status Solidi A 190, 135 (2002).

${ }^{19}$ E. Matioli, S. Keller, F. Wu, Y.-S. Choi, E. Hu, J. Speck, and C. Weisbuch, J. Appl. Phys. 106, 024309 (2009).

${ }^{20}$ E. Matioli, E. Rangel, M. Iza, B. Fleury, N. Pfaff, J. Speck, E. Hu, and C. Weisbuch, Appl. Phys. Lett. 96, 031108 (2010). 